\title{
Analysis of TLM Air-vent Model Applicability to EMC Problems for Normal Incident Plane Wave
}

\author{
Nataša J. Nešić and Nebojša S. Dončov, Member, IEEE
}

\begin{abstract}
In this paper, the shielding properties related to a protective metal enclosure with airflow aperture arrays are numerically analyzed. As a numerical model, a TLM method, either in a conventional form based on fine mesh to describe apertures presence or enhanced with the compact air-vent model is employed. The main focus in the paper is on examining the limits of applying the compact air-vent model for EMC problems solving. Namely, various values for the distance between neighboring apertures in the TLM air-vent models as well as the air-vent thicknesses are analyzed. Specifically, the analyses are conducted for a normal incident plane wave, vertically and horizontally polarized.
\end{abstract}

Keywords - Enclosure, fine TLM mesh, hexagonal apertures, plane wave, round apertures, shielding effectiveness, TLM Air-vent model.

\section{INTRODUCTION}

$\mathrm{O}$ $\mathrm{NE}$ of the major challenges in the design of an electronic system is to harmonize electromagnetic (EM) radiation and eliminate potential electromagnetic interferences (EMI). In order to protect electronic circuits from some external influences, they are often put in some shielding enclosures, which are made of materials to a wide range of electrical conductivity, magnetic permeability, and thickness [1]. However, the shields commonly contain some apertures, slots and/or seams which enable an external EM radiation to penetrate inside, and which in turn results in distortion of their protective function. The focus of this paper is put on the enclosures for high-speed digital designs that use airflow aperture arrays (so called air-vents), instead of large open

Paper received May 30, 2016; revised October 7, 2016; accepted October 10, 2016. Date of publication November 20, 2016. The associate editor coordinating the review of this manuscript and approving it for publication was Prof. Branko Kolundžija.

This paper is a revised and expanded version of the paper presented at the 23rd Telecommunications Forum TELFOR 2015 [14].

This work has been partially supported by the Ministry for Education, Science and Technological Development of Serbia, project number TR32052 and EUROWEB+ project.

Corresponding Nataša $J$. Nešić is with the College of Applied Technical Sciences, Niš, Aleksandra Medvedeva 20, 18000 Niš, Serbia (phone: 381-64-2341790; e-mail: natasa.bogdanovic@vtsnis.edu.rs).

Nebojša S. Dončov is with the Faculty of Electronic Engineering in Niš, University of Niš, Aleksandra Medvedeva 14, 18000 Niš, Serbia; (phone: 381-18-529638, e-mail: nebojsa.doncov@elfak.ni.ac.rs). apertures, for ventilation and heat dissipation [2]. Specifically, here we examine the shielding properties of an enclosure with such apertures from the viewpoint of shielding effectiveness (SE).

There is a number of numerical techniques for computational analysis of various EM and electromagnetic compatibility (EMC) problems. Probably the most popular methods among the differential time-domain ones are the finite-difference time-domain (FD-TD) method [3] and the transmission line matrix (TLM) technique [4]. The TLM method has been already enhanced in order to efficiently account for small but influential features instead of using a fine mesh to model their presence [4-8]. One of these socalled sub-cell models is presented in [9-11] to permit modeling of air-vents on thin and thick enclosure metal walls. The accuracy of this compact air-vent model has been successfully verified through comparison with experimental [9] and fine TLM mesh results [9,10]. Also, the efficiency of this model has been demonstrated on several classical EMC problems examples [11]. However, note that the applicability of the compact TLM air-vent model to EMC problems has a few possible limitations [9$11]$.

In this article, we investigate the effects of arbitrary distance between apertures and different perforated wall thicknesses from the TLM air-vent model applicability point of view. The analysis is done for both a horizontal and vertical incident plane wave.

\section{BRIEF DESCRIPTION OF TLM AIR-VENT MODEL}

An efficient numerical calculation of EM field inside protective enclosures and estimation of its shielding properties can be accomplished by using a TLM method of compact air-vent model. The compact TLM air-vent model consists of two reactive circuits per propagation direction, which are placed between two neighboring TLM cells. The TLM cells coincide with the position of perforated metal wall, as illustrated in Fig. 1. Firstly, it was developed for square and circular perforations on thin metal walls [9] and on walls of significant thickness [10]. Afterwards, it was extended for air-vents with rectangular and hexagonal apertures [11].

To capture both horizontally and vertically polarized EM field propagation through the perforations, two reactive circuits are needed. Each of them interacts with voltage pulses travelling through one of two orthogonally polarized link lines of TLM cell. Note that the apertures 


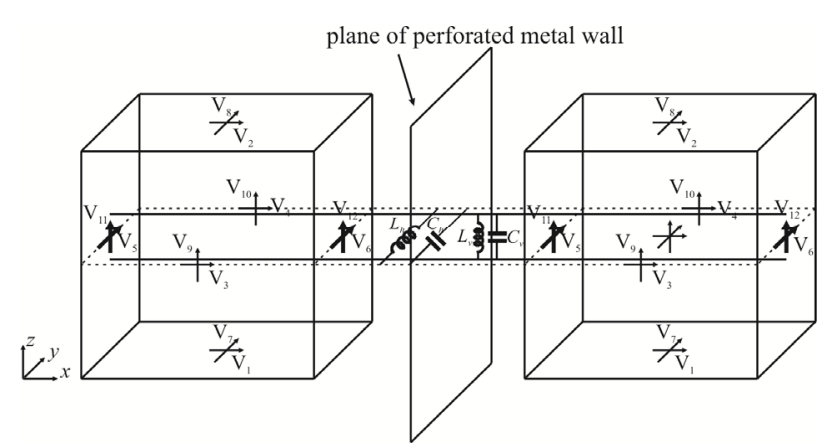

Fig.1 Compact TLM air-vent model of perforated metal wall in $y z$ plane; $L_{h} C_{h}$ circuit for horizontal and $L_{v} C_{v}$ circuit for vertical polarization.

should be analysed in two dimensions. Each circuit is current running near the aperture borders, while the latter one models the EM field distribution inside the apertures. The equivalent reactance, denoted by $X$, can be empirically found by a series of TLM fine mesh simulations conducted for a plane wave illuminating perforated metal wall of different thicknesses and apertures' mutual distances. It is expressed as a product of angular frequency and four other terms that depend on aperture cross-section form, cut-off frequency of the aperture $f_{c}$, coverage cov (percentage of wall surface covered by apertures) and wall thickness $t$ or perforation depth [11]:

$$
\begin{aligned}
& X=\omega^{*} A_{0} *\left(\frac{Z_{0} / 2}{2 \pi f_{\mathrm{c}}}\right) * \\
& \left(A_{1} \operatorname{cov}+A_{2} \operatorname{cov}^{2}+A_{3} \operatorname{cov}^{3}\right) * \operatorname{Exp}\left(-A_{4} \frac{t}{v /\left(2 \pi f_{\mathrm{c}}\right)}\right) .
\end{aligned}
$$

Constants $A_{i}, \mathrm{i}=1,2,3,4$ in (1) are given in [11] for each considered aperture shape and appropriate polarization. In case where the propagation of an incident plane wave, irrespectively of its polarization, can be represented by an equivalent circuit consisting of a line of characteristic impedance $Z_{0}$ loaded with reactance $X$ inserted at its midpoint, the transmission coefficient is given by the following expression [9-11]:

$$
T=j X /\left(j X+Z_{0} / 2\right) \text {. }
$$

The reactance $X$, i.e. its capacitance and inductance in parallel are selected so as to match the value of transmission coefficient at two different frequencies corresponding to $10 \%$ and $80 \%$ of aperture cut-off frequency.

The main advantage of compact TLM air-vent model is that a coarse mesh can be used with cells that can be potentially bigger in size than individual apertures. This stands in sharp contrast with the fine mesh modeling where a number of cells are necessary across each aperture dimension. Also, there is no need to model aperture depth as it is included explicitly in the model through parameter $t$ (thickness).

However, the TLM air-vent model has several drawbacks when used for EMC problems. Some of them are obvious; for example, there are no structures beyond a perforation as this will obviously not correspond to the way the transmission coefficient is calculated in (2). Also, the model is applicable to the frequencies below the cut- off frequency of the perforation as we consider aperture on a metal wall as an extremely short, highly cut-off waveguide. Other model limitations are less obvious; for instance, the applicability of the model is limited to frequencies at which perforations are closer than a half wavelength apart [12]. Also, model is valid within the limits of defined parameters which in the case of perforation depth go from indefinitely thin perforated metal wall to the wall with thickness up to the crosssectional dimension of the aperture. Perforated walls with extremely small coverage are also not taken into account during the empirical derivation of compact model. Some of these limits will be studied in the next section.

\section{NUMERICAL ANALYSIS AND RESULTS}

This section is devoted to the numerical results related to the shielding effectiveness of a protective metal enclosure with circular perforations. The results are obtained by employing a fine mesh and a coarse mesh with the compact air-vent model which has been described in the previous section. In order to investigate the applicability limits of the compact air-vent model, different values for both the distances between circular apertures and the wall thickness are examined.

\section{A. Numerical Models}

Let us consider a protective metal enclosure with circular perforations on its frontal wall, as depicted in Fig. 2(a). We will first model the enclosure by using a conventional TLM method based on applying a fine mesh to describe the apertures. The enclosure is of a rectangular shape, with inner dimensions $(100 \times 100 \times 200) \mathrm{mm}$. Also, it has eight round apertures, where each aperture is of a diameter of $12.77 \mathrm{~mm}$. The apertures are positioned symmetrically around the center on the frontal wall. The horizontal and vertical spacing between any two apertures is $d=2 \mathrm{~mm}$, see Fig. 2(c). The wall thickness of $t=2 \mathrm{~mm}$ is constant for the whole enclosure. In order to accurately model the enclosure's thickness and its circular apertures, the mesh with fineness of 26 cells along $z$ and $x$ axes is used to model each aperture, while 4 cells are used to describe the apertures mutual distance and the wall thickness. A plane wave of the vertical polarization with the propagation in $y$-direction and with the electric field component in $z$-direction is used as an excitation. The characteristics of the enclosure, such as its geometry, dimensions and shape of apertures, as well as the excitation are assumed to be as in [13].

Next, the same enclosure is also modeled by employing the coarse mesh with incorporated compact air-vent model. The part of frontal wall on which the model is applied is of dimensions $a^{\prime}=29.54 \mathrm{~mm}$ and $b^{\prime}=59.08$ $\mathrm{mm}$, where $a^{\prime}$ and $b^{\prime}$ are depicted in Fig. 2(c). These dimensions are determined based on the rule that the apertures are at half mutual distance $(d / 2)$ from the modeled part border. To describe this area, the number of cells used along $x$ axis equals 10, while 20 cells are used along $z$ axis. The percentage of the surface of this part of frontal wall covered by apertures is $58.71 \%$. 


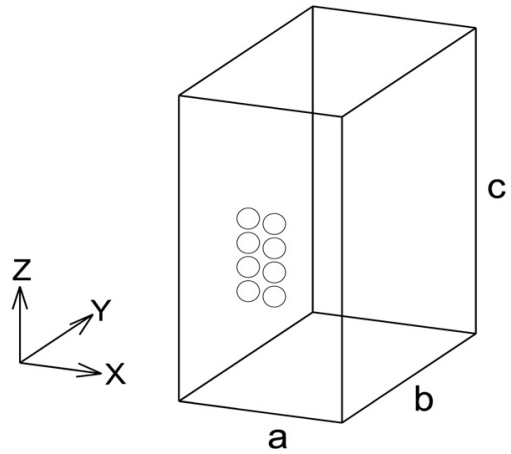

a)

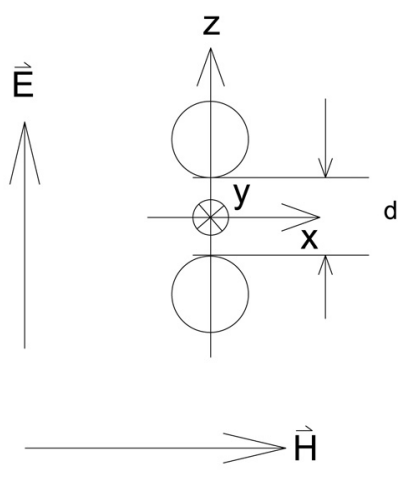

b)

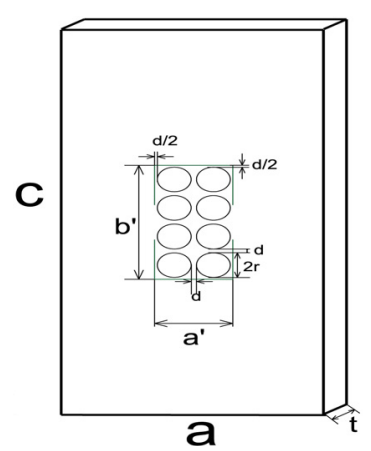

c)
Fig 2 (a) Protective metal enclosure of rectangular cross-section, (b) Two apertures along the $z$-axis,

c) Frontal wall with ventilation apertures.

Figure 3 presents the results for the enclosure SE obtained by previously described fine mesh and coarse mesh with incorporated compact air-vent model in comparison with the SE curve from reference [13]. Observe that the figure shows a very good agreement between the first two curves in the whole analyzed frequency range, which is from 0 up to $3 \mathrm{GHz}$. Also, it can be noted that the SE levels in both cases are slightly higher at lower frequencies in comparison with the SE measurement curve from [13]. Moreover, the first two resonant frequencies are shifted toward lower frequencies in contrast to the SE curve in [13]. The differences can be explained by the fact that the inner space of the enclosure is slightly larger than in [13] due to differently defined internal dimensions of enclosure.

Next, the expression for the resonant frequencies of rectangular enclosure $f_{m n l}$ is provided below:

$$
f_{m n l}=\frac{c_{0}}{2 \pi \sqrt{\varepsilon_{r} \mu_{r}}} \sqrt{\left(\frac{m \pi}{a}\right)^{2}+\left(\frac{n \pi}{b}\right)^{2}+\left(\frac{l \pi}{c}\right)^{2}} .
$$

where $a, b$, and $c$ are dimensions of a rectangular enclosure, $c_{0}$ is the velocity of light in free-space, $\varepsilon_{r}$ and $\mu_{r}$ are relative permittivity and relative permeability, and $m, n$, and $l$ are integers where at least two of $\{m, n, l\}$ must be greater than zero. The resonant frequencies of $\mathrm{TE}_{110}$ and $\mathrm{TE}_{112}$ cavity modes, analytically calculated by using the expression in (3), for the enclosure dimensions which are previously given, are at $2.121 \mathrm{GHz}$ and $2.598 \mathrm{GHz}$, respectively. The values of these two resonances, obtained by the compact air-vent model, are at $2.119 \mathrm{GHz}$ and $2.583 \mathrm{GHz}$, are in excellent agreement with the ones obtained by the fine mesh $(2.118 \mathrm{GHz}$ and $2.584 \mathrm{GHz})$. It should be mentioned that the resonance values for both models are closer to the analytical values than the measurement results, which are equal to $2.210 \mathrm{GHz}$ and $2.668 \mathrm{GHz}$.

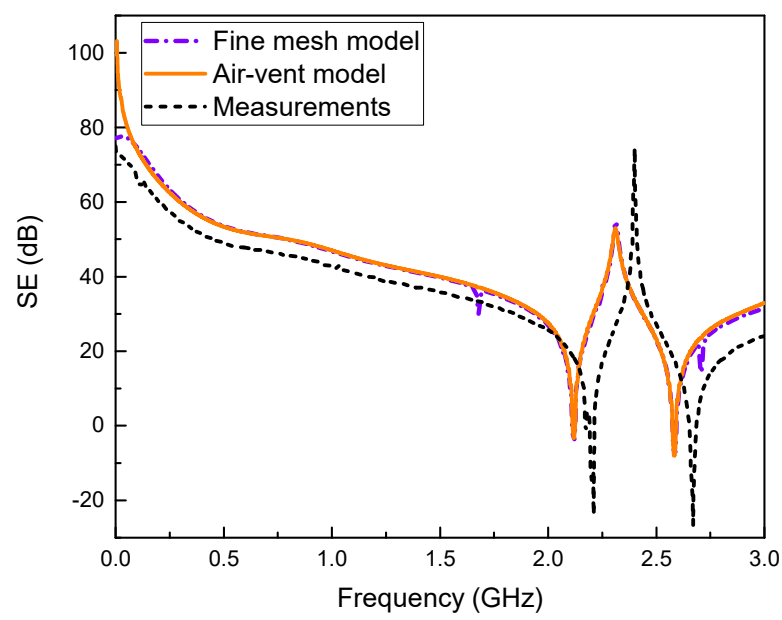

Fig. 3 The SE curves of enclosure with an array of 8 round apertures $(d=2 \mathrm{~mm})$ compared to measurements [13] for vertical polarization.

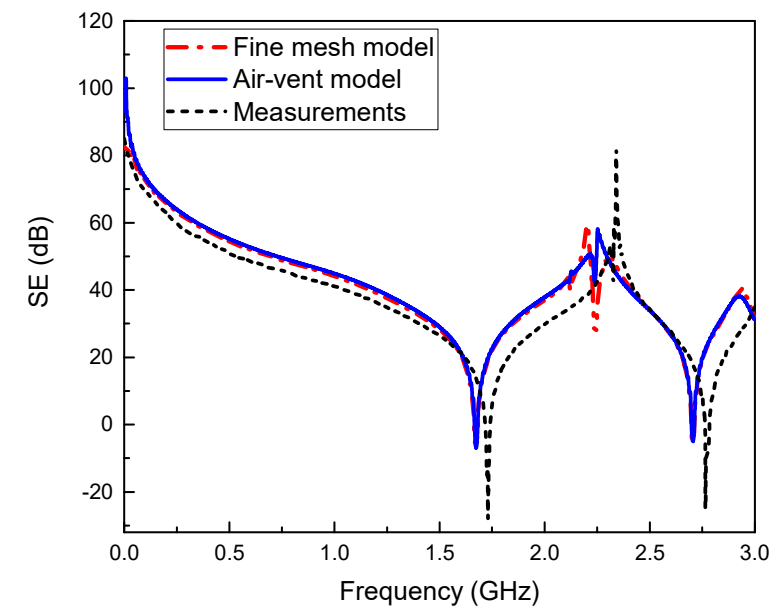

Fig. 4 The SE curves of enclosure with an array of 8 round apertures $(d=2 \mathrm{~mm})$ compared to measurements

[13] for horizontal polarization.

In Fig. 4 a similar comparison is performed; now, a horizontally polarized plane wave is used instead. The SE levels obtained by the fine mesh and the air-vent model show an excellent agreement. Here, the resonant frequencies of $\mathrm{TE}_{011}$ and $\mathrm{TE}_{013}$ cavity modes, analytically calculated from (3) for horizontal polarization, are at the frequency values of $1.677 \mathrm{GHz}$ and $2.704 \mathrm{GHz}$, respectively. These two resonant frequencies obtained by the air-vent model are equal to $1.674 \mathrm{GHz}$ and $2.705 \mathrm{GHz}$. In the fine mesh scenario, these resonances take the values of $1.673 \mathrm{GHz}$ and $2.705 \mathrm{GHz}$, respectively. In addition to this, a very good resonance fit for both numerical models is obtained. Again, the resonances have a small shift toward the lower frequencies compared to the SE curve in [13] (in which the resonances are at $1.730 \mathrm{GHz}$ and 2.765 $\mathrm{GHz}$ ) due to the same reasons as explained before. 


\section{B. Aperture spacing}

In this subsection, we change the aperture spacing while using the same enclosure as in subsection A. We firstly set the spacing to be $18 \mathrm{~mm}$ and compare the fine mesh and the air-vent model, see Fig. 5. The figure indicates that the SE curve related to the enclosure modeled with the fine mesh has a similar frequency offset at the first two resonant frequencies, with respect to the one obtained by compact air-vent model, as in the case where the aperture spacing of $2 \mathrm{~mm}$ was used. When analyzing the SE levels, it can be noticed that the SE curves for both models are in a good mutual agreement in the whole observed frequency range.

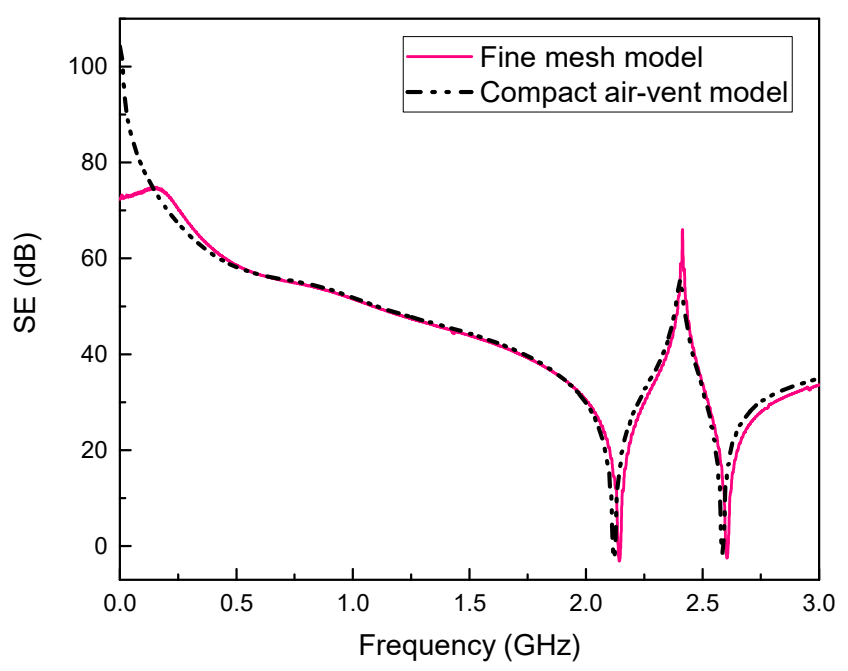

Fig. 5 The SE curves of enclosure with an array of 8 round apertures $(d=18 \mathrm{~mm})$ - vertical polarization.

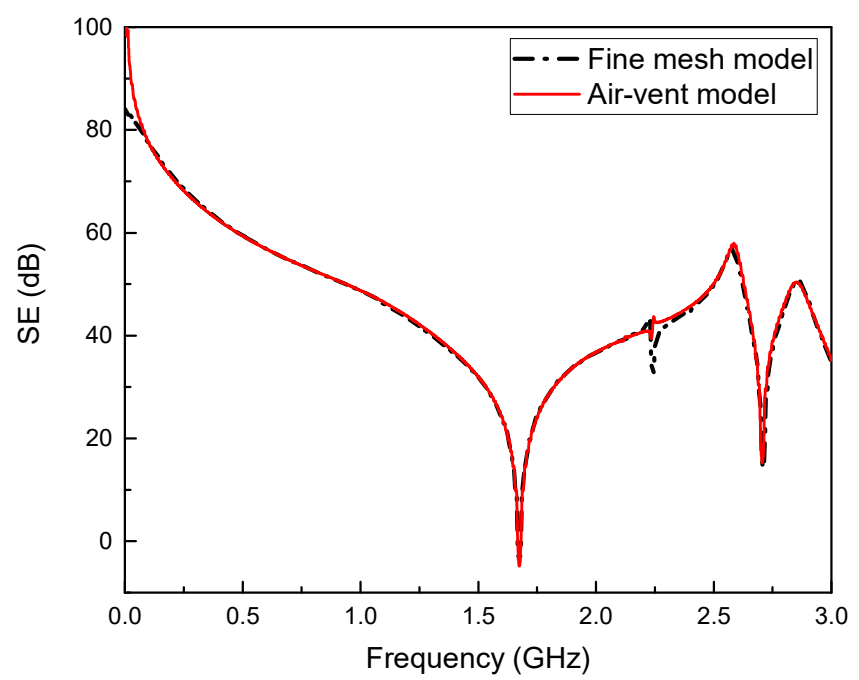

Fig. 6 The SE curves of enclosure with an array of 8 round apertures $(d=18 \mathrm{~mm})$ - horizontal polarization.

The same aperture distance of $18 \mathrm{~mm}$ for both numerical models is performed. Now, the enclosure is excited by a horizontally polarized plane wave. An excellent mutual match between both SE curves can be observed, with the exception in a small peak which occurs at frequency above $2 \mathrm{GHz}$, as depicted in Fig. 6.

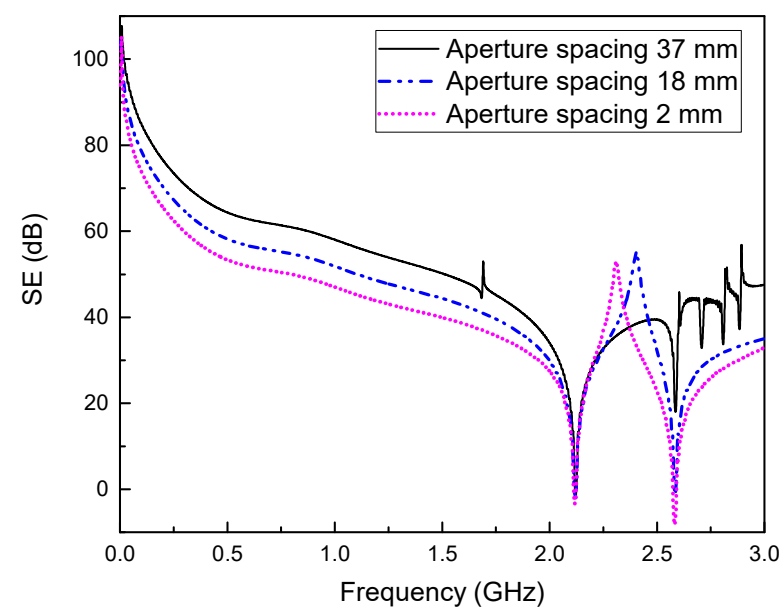

Fig. 7 The SE curves of enclosure with circular airvents for different spacing - vertical polarization

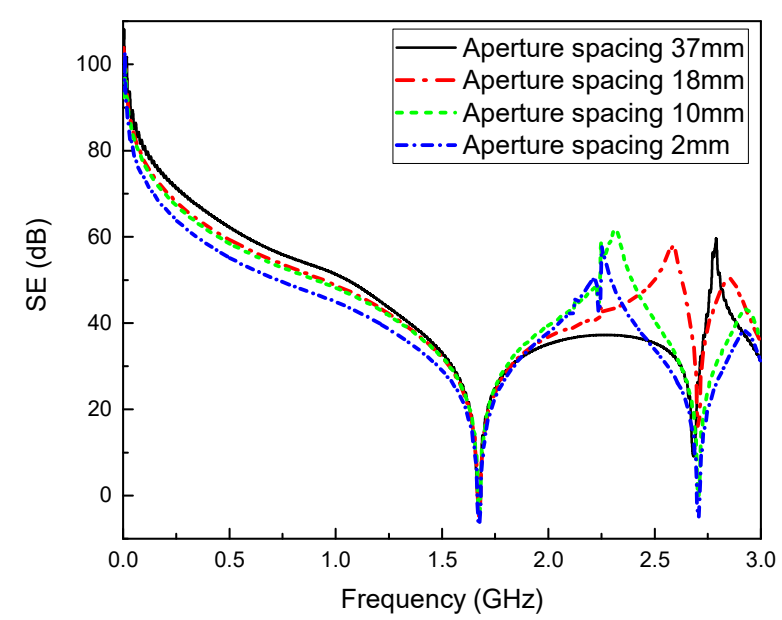

Fig. 8 The SE curves of enclosure with circular airvents for different spacing - horizontal polarization.

We proceed now by considering the case in which the aperture spacing is increased to the value of $37 \mathrm{~mm}$. The analysis is now done only for the air-vent model and the results are compared to the ones for the aperture spacing of $18 \mathrm{~mm}$ and $2 \mathrm{~mm}$. As Fig. 7 illustrates, the values of the first two resonant frequencies, which are taking the values of $2.119 \mathrm{GHz}$ and $2.583 \mathrm{GHz}$, do not change with the increase of aperture spacing. However, at a given frequency, the SE level values corresponding to the three analyzed cases differ in general due to the fact that an increase of aperture spacing (which also increases the dimensions of the part of the wall on which the air-vent model is applied) causes that the coverage decreases. Namely, the frontal wall covered by the apertures with distances of $18 \mathrm{~mm}$ and $37 \mathrm{~mm}$ is $13.52 \%$ and $5.17 \%$, respectively. Most importantly, additional (vibrant) resonant peaks at higher frequencies appear due to the fact that air-vent model cannot give good results at frequencies higher than $2.5 \mathrm{GHz}$.

In the same air-vent model, the same enclosure is excited by a horizontally polarized plane wave. The aperture space of air-vents is set to be at distances of 2 $\mathrm{mm}, 10 \mathrm{~mm}, 18 \mathrm{~mm}$ and $37 \mathrm{~mm}$. The distance between apertures of $d=37 \mathrm{~mm}$ has the coverage of $5.17 \%$. The SE results are compared with the others of various aperture distances, such as $2 \mathrm{~mm}, 10 \mathrm{~mm} 18 \mathrm{~mm}$ and 37 
$\mathrm{mm}$, as illustrated in Fig. 8. It can be noticed that, although the resonances are the same, with the increase in aperture distance the SE levels are slightly higher up to the first resonant frequency. After that, all the curves have different forms. Moreover, the model still works for the aperture spacing of $37 \mathrm{~mm}$, which can be said to be the border of the model applicability. In addition, by calculating the coverage of air-vents, which should be at distance of $d=38 \mathrm{~mm}$, the parameters $a^{\prime}$ and $b^{\prime}$ are exceeded the physical enclosure dimensions, $101.54 \mathrm{~mm}$ and $203.08 \mathrm{~mm}$, respectively. If there is a need to satisfy the requirement that the enclosure should have an air-vent with 8 perforations, the distance of $37 \mathrm{~mm}$ meets the requirement and it is set to be the limit of the model applicability.

In the following, the same enclosure with hexagonal air-vents for the plane wave of vertical polarization is conducted. The enclosure has eight hexagonal apertures with the length side of $l=7.02 \mathrm{~mm}$. Firstly, the aperture space between any two apertures along $x$-axis and $z$-axis of $2 \mathrm{~mm}$ is analyzed. For that case, the frontal enclosure wall on which the hexagonal air-vents exist has the percentage of surface area covered by apertures of 56.37 $\%$. Further, we set the aperture spacing to be $18 \mathrm{~mm}$ and the coverage of $13.25 \%$ in order to obtain the SE characteristics. Finally, the aperture distance is put on 36 $\mathrm{mm}$ to examine the applicability of the model for the very small coverage, such as $5.32 \%$. The SE curves for different coverage are illustrated in Fig. 9. It can be noted that the values of the resonances, which are around 2.120 $\mathrm{GHz}$ and $2.587 \mathrm{GHz}$, do not change by increasing the distances between apertures. The SE levels increase with reducing the coverage of perforated wall. But the air-vent model does not give correct results for an aperture spacing of $36 \mathrm{~mm}$. Especially, additional peaks appear at higher frequencies above $2.5 \mathrm{GHz}$, as depicted in Fig 9 .

In the case of circular and hexagonal air-vents, for the similar air-vent coverage and the same type of polarization, the SE characteristics have similar values.

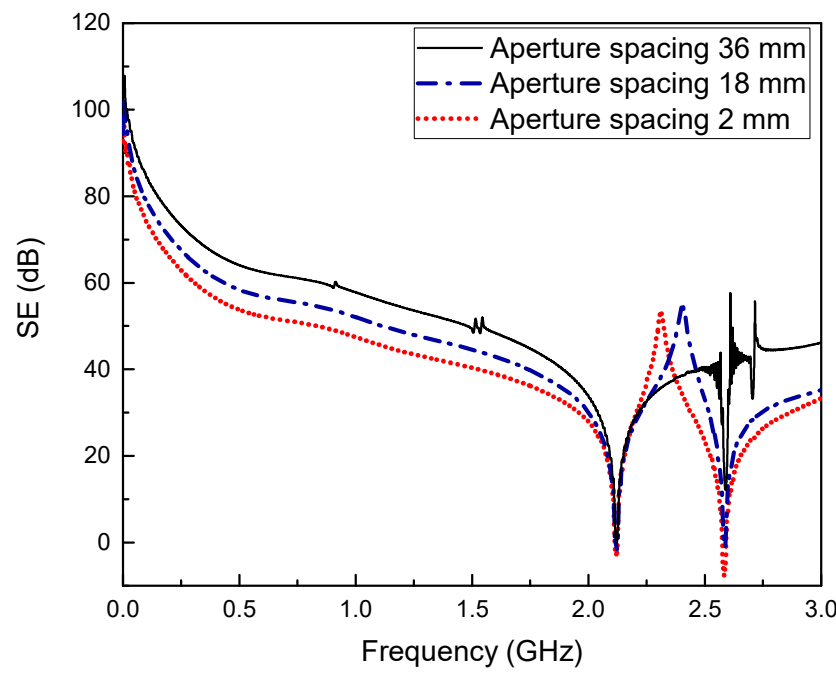

Fig. 9 The SE curves of enclosure with an array of 8 hexagonal apertures for different spacing - vertical polarization.

\section{Wall thickness}

In order to test an air-vent model in a case of a thick perforated panel with thickness greater than the crosssectional dimension of the aperture, different thicknesses of frontal wall from Fig. 2(c) are considered next $(2 \mathrm{~mm}$; $10 \mathrm{~mm} ; 20 \mathrm{~mm} ; 50 \mathrm{~mm})$. The last two values are greater than the diameter of circular apertures in the air-vent screen. The distance between apertures is assumed to be 2 $\mathrm{mm}$ and coverage is calculated accordingly. The results for the SE of enclosure, obtained by the air-vent model, for all considered thicknesses are shown in Fig. 10. It can be seen that the shape of SE curve remains the same regardless of frontal wall thickness. Also, the relative ratio between two adjacent SE curves is almost constant in the considered frequency range and follows the value obtained when empirical formula of $32 *(t / 2 r) \mathrm{dB}$ [2] is applied for each thickness in order to correct its SE level with the respect of infinity thin perforated panel.

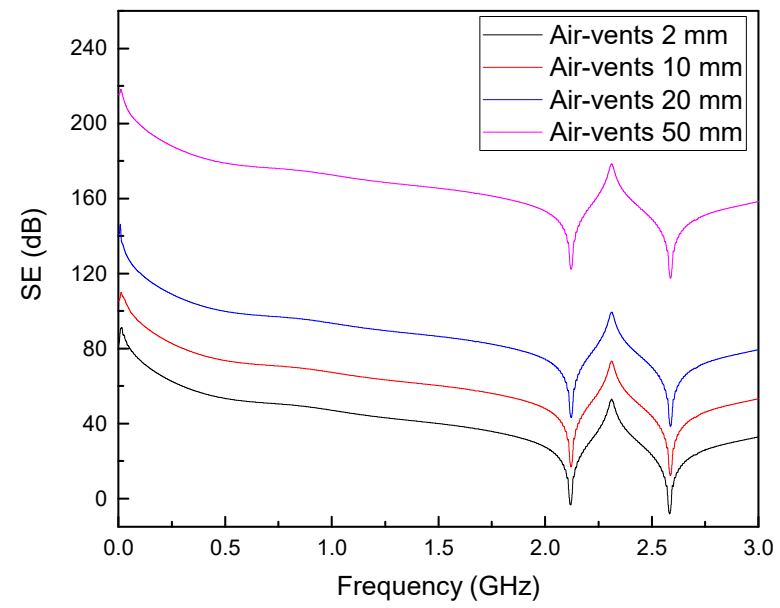

Fig. 10 The SE curves for the cases of different depths of frontal wall covered by circular air-vents - vertical polarization.

For example, the relative ratio between the SE values of two curves in Fig. 10, obtained for thicknesses of $10 \mathrm{~mm}$ and $20 \mathrm{~mm}$, is around $20 \mathrm{~dB}$ which corresponds to a difference of $25.1 \mathrm{~dB}$ and $5 \mathrm{~dB}$ calculated when empirical formula from [2] is applied to each thickness, see Table 1. Therefore, it can be said that the air-vent model is able to give correct results even in cases when perforation depth is outside the range for which the model is developed.

The effect of different thickness values on the air-vent model when excited by a horizontally polarized plane wave is analyzed next. The thickness values of $2 \mathrm{~mm}, 10$ $\mathrm{mm}$ and $15 \mathrm{~mm}$ are considered and the SE results are presented in Fig. 11. It can be noticed that the SE characteristics are of the same shape while there are significant differences in their levels. Figure 11 presents, for instance, that the SE characteristics of air-vents thicknesses of $10 \mathrm{~mm}$ and $15 \mathrm{~mm}$ differ about $12 \mathrm{~dB}$ which is in accordance with the level difference between $37.59 \mathrm{~dB}$ and $25.1 \mathrm{~dB}$ given by empirical formula [2]. Also, the SE values for different enclosure thicknesses are given in Table 2. 


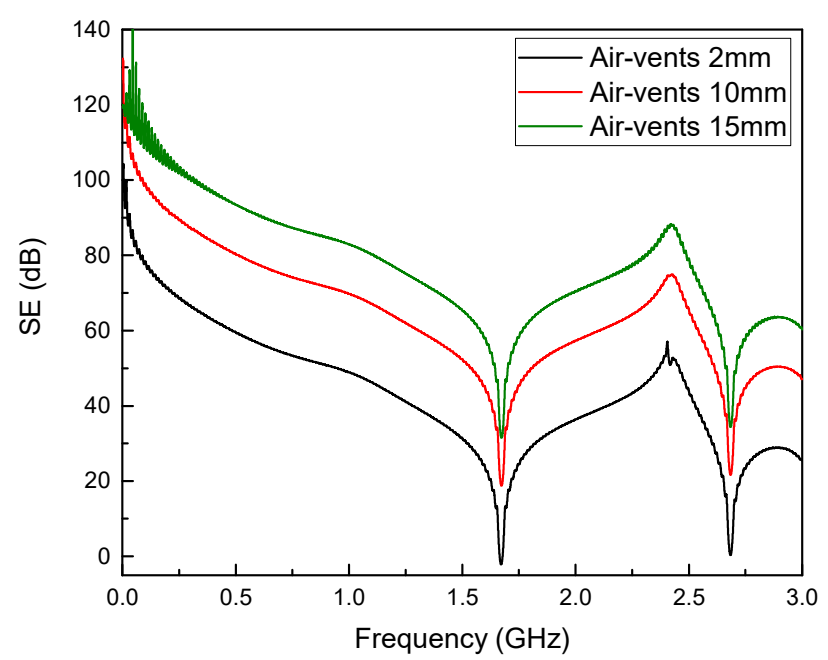

Fig. 11 The SE curves for the cases of different depths of frontal wall covered by air-vents - horizontal polarization.

TABLE 1: SE LEVEL CORRECTION

\begin{tabular}{l|r}
\hline $\begin{array}{l}\text { Depth of air-vent hole [mm] } \\
\text { vertical polarization }\end{array}$ & $\begin{array}{r}\text { Empirical } \\
\text { formula [2] }\end{array}$ \\
\hline 2 & 5 \\
10 & 25.1 \\
20 & 50.1 \\
50 & 125.3 \\
\hline
\end{tabular}

TABLE 2: SE LEVEL CORRECTION

\begin{tabular}{l|r}
\hline $\begin{array}{l}\text { Depth of air-vent hole [mm] } \\
\text { horizontal polarization }\end{array}$ & $\begin{array}{r}\text { Empirical } \\
\text { formula [2] }\end{array}$ \\
\hline 2 & 5 \\
10 & 25.1 \\
15 & 37.59 \\
\hline
\end{tabular}

\section{CONCLUSION}

In this paper, the TLM air-vent model has been tested at the frequencies at which the aperture spacing is greater than a half of the wavelength and for perforated wall thicknesses bigger than apertures diameter. Also, analyses are conducted for the normal incident plane wave of both vertical and horizontal polarization. The TLM air-vent models with round and hexagonal apertures are analyzed for different values of the spacing between the apertures. The results have shown that, with an aperture spacing increase, the resonant frequencies remain the same while some additional resonant peaks may appear at higher frequencies. Afterwards, the effect of an increase of the wall thickness has been examined and it has been shown that the levels are higher. Future research will be focused on the investigation of other possible model limits, such as for an oblique incident plane wave and perforated walls with extremely small coverage, and on finding solutions to overcome identified limits.

\section{REFERENCES}

[1] C. Christopoulos, Principles and Techniques of Electromagnetic Compatibility, 2nd ed., CRS Press, 2007.

[2] M. Li, J. Nuebel, J. L. Drewniak, R. E. DuBroff, T. H. Hubing, T. P. Van Doren, "EMI from Airflow Aperture Arrays in Shielding Enclosures-Experiments, FDTD, and MoM Modelling," IEEE Trans. on Electromagn. Compat., vol. 42, no. 3, pp. 265-2275, 2000.

[3] K. S. Kunz, R. J. Luebbers, "The Finite Difference Time Domain Method for Electromagnetics," CRC Press, Boca Raton, FL, 1993.

[4] C. Christopoulos, The Transmission-Line Modelling (TLM) Method, IEEE/OUP Series, Piscataway, NJ, 1995.

[5] A. J. Wlodarczyk, V. Trenkic, R. Scaramuzza, C. Christopoulos, "A fully Integrated Multiconductor Model for TLM," IEEE Trans. Microwave Theory Tech., vol. 46, no. 12 pp. 2431-2437, 1998.

[6] J. Paul, C. Christopoulos, D.W.P. Thomas, X. Liu, "Time-domain modeling of electromagnetic wave interaction with thin-wires using TLM," IEEE Trans. Electromagn. Compat., vol. 47,no. 3, pp. $447-$ 455,2005

[7] V. Trenkic, R. Scaramuzza, "Modelling of arbitrary slot structures using transmission line matrix (TLM) method," Int. Symp. Electromagn. Compat., Zurich, Switzerland, 2001, pp. 393-396.

[8] J. Paul, V. Podlozny, C. Christopoulos, "The use of digital filtering techniques for the simulation of fine features in EMC problems solved in the time Domain," IEEE Trans. Electromagn. Compat., vol. 45 , no. 2 , pp. 238-244, 2003.

[9] N. Doncov, A. J. Wlodarczyk, R. Scaramuzza, V. Trenkic, "TLM modelling of perforated metal screens," 4th Int. Conf. Computational Electromagnetics, Bournemouth, UK, 2002.

[10] N. Doncov, A. J. Wlodarczyk, R. Scaramuzza, V. Trenkic, "Compact TLM model of air-vents," Electronics Letters, vol. 38, no. 16, pp. 887-888, Jan. 2002.

[11] N. Doncov, B. Milovanovic, Z. Stankovic, "Extension of compact TLM air-vent model on rectangular and hexagonal apertures," ACES Journal, vol. 26. No.1, pp. 64-72, 2011.

[12] N. Dončov, "Compact TLM Models for Efficient EMC Simulations," presented at the 6th Int. Conf. on Telecommunications in Modern Satellite, Cable and Broadcasting Service: Serbia and Montenegro, Niš, 1-3 October, 2003, pp. 461470, 0-7803-7963-2/03/\$17.00 (C2003 IEEE.

[13] BL. Nie, PA. Du, YT. Yu, Z. Shi, "Study of the Shielding of Enclosure with Apertures at Higher Frequencies Using the Transmission-Line Modeling Method," IEEE Trans. on Electromagn. Compat., vol. 53. No.1, pp. 73-81, Feb. 2011.

[14] N. J. Nešić and N. S. Dončov, "Analysis of TLM air-vent model applicability to EMC problems," Telecommunications Forum Telfor (TELFOR), 2015 23rd, Belgrade, 2015, pp. 579-582. 